\title{
Numerical Investigation into the Two-Phase Convective Heat Transfer within the Hold of an Oil Tanker Subjected to a Rolling Motion
}

\author{
Guojun Yu ${ }^{1,2, *\left(\mathbb{D}, \text { Sheng } \mathrm{Jia}^{1} \text { and Yanting Geng }\right.}{ }^{1}$ \\ 1 Merchant Marine College, Shanghai Maritime University, Shanghai 201306, China; \\ 201730110052@stu.shmtu.edu.cn (S.J.); 201610129071@stu.shmtu.edu.cn (Y.G.) \\ 2 School of Naval Architecture, Ocean \& Civil Engineering, Shanghai Jiao Tong University, \\ Shanghai 200240, China \\ * Correspondence: gjyu@shmtu.edu.cn
}

Received: 20 March 2019; Accepted: 1 April 2019; Published: 3 April 2019

check for updates

\begin{abstract}
A crude oil tanker usually encounters a rolling motion during sea transportation, which leads to rotational movement and sometimes a sloshing of the liquid hold. This rolling-induced body motion seriously affects the thermal and hydraulic behavior of the liquid hold, which then affects the heating process and heat preservation of the tanker. Clarification of the involved thermal and hydraulic characteristics is the basic requirement for establishment of a scientific heating scheme and heat preservation method. A two-phase 3D model considering the free liquid surface and non-Newtonian behavior of the fluid was established for the thermal calculation of the liquid holds in oil tankers. The thermal and hydraulic characteristics of the liquid hold were investigated under different combinations of dimensionless parameters, and the combined effect of rolling and fluid non-Newtonian behavior was investigated. It was found that rolling intensifies the heat transfer based on the combination of the Richardson number $(R i)$ and the rotation-strength number $\left(\omega^{*}\right)$, and non-Newtonian behavior of the fluid effectively affects the heat transfer in a rolling motion. This research is expected to provide a reference for design and optimization of the heating and heat preservation method for oil tanker operation.
\end{abstract}

Keywords: oil tanker; rolling motion; mixed convection

\section{Introduction}

Tanker transportation is one of the most important ways for crude oil transportation, especially during international crude oil trade. Some high-pour point or high-viscosity crude oil requires heating during transportation to ensure good flow ability for flexible offloading. This heating process consumes large amounts of energy, and a scientific heating scheme bears great potential to save energy. However, such a scientific heating scheme is based on the full understanding of the thermal and hydraulic behavior of the crude oil in the ocean condition. The thermal-hydraulic behavior of crude oil in ocean conditions is much more complicated than that in static conditions, since the flow and heat transfer is influenced by the motion-induced additional forces, such as centrifugal force, Euler force, Coriolis force, and buoyancy forces induced by these forces, in addition to the forces for static cases. The motion of the tanker during sea transportation includes oscillations of six degrees of freedom, such as linear motions, including heaving, swaying, and surging, and rotational motions, including rolling, pitching, and yawing. Among these movements, the rolling motion has a more important influence on the thermal-hydraulic behavior [1]. Therefore, only the rolling motion is investigated in this research.

There are many studies regarding the effect of a rolling motion on flow and heat transfer within oil tankers in the open literature. Hiroharu [2] studied the heat transfer characteristic of oil in a 1/50 
scale model of a tanker in a rolling condition, finding that oil temperature in the tanker becomes uniform with rolling motion, and the heat transfer coefficient increases with increasing of amplitude and frequency of rolling. Yu et al. [3] studied the temperature drop of an oil tanker with a free liquid surface subjected to a rolling motion, indicating that the heat transfer of oil is dominated by mixed convection rather than natural convection under a rolling condition, and the temperature drop rate increases with the oscillation frequency. Similarly, only a small-size model tanker was investigated. Doerffer et al. [4] studied the influence of low-frequency harmonic oscillation on the natural convection occurring at the vertical walls of a tanker, pointing out that the dimensionless group $G r /\left(\operatorname{Re}^{2} \operatorname{Pr}^{1 / 3}\right)$ (Gr, Re, Pr are Grashof number, Reynolds number and Prandtl Number respectively) can be regarded as the principal criterion for this kind of mixed convection and it decreases corresponding to the increasing participation of forced convection in mixed convection. Akagi et al. [5] studied the mixed convection heat transfer in an oil tanker subjected to a rolling motion, taking the inertia forces, including the centrifugal force and Coriolis force into consideration, indicating that forced convection plays an important role during a rolling motion.

Similar research can also be found in the area of convection heat transfer in pipe flow or channel flow. Most of these studies also indicated that the rolling affected the thermal-hydraulic behavior. Tan et al. [6,7] studied single-phase natural circulation heat transfer in a pipe system with a rolling motion. They found that the fluid flow fluctuates periodically due to the rolling motion, and the heat transfer coefficient increases with the rolling amplitude and frequency. Yu et al. [8] studied the fluid temperature fluctuation at the outlet of the test section in a single-phase natural circulation loop with a heat narrow rectangular channel under rolling motion conditions, indicating that the average temperature of fluid at the outlet of the test section under rolling motion conditions is higher than that under static conditions because of the decreasing cycle averaged flow rate. In addition, Liu et al. [9] studied the safety boundary of flow instability and critical heat flux for parallel channels in static and ship motion. Wu et al. [10] studied the thermal-hydraulic behavior of a nuclear reactor core in an ocean environment. Xing et al. [11] studied the effects of ocean conditions on coolant flow in nuclear power systems in sea transportation. Besides, Hu et al. [12] studied the hydraulic dynamics behavior in floating LNG (Liquefied Natural Gas) systems under ocean conditions. Grotle et al. [13] studied the thermal response in marine LNG fuel tanks by experiments and modelling. Jiang et al. [14] analyzed the effect between ship motion response and internal sloshing flow. Cercos-Pita [15] investigated the coupled nonlinear dynamics of a vessel with a free surface tank onboard. All this research indicates rolling has obvious effects on flow and heat transfer.

It can be found from the above literature review that there are many researchers focusing on the heat and flow characteristic of fluid in a rolling system, but their models are restricted to either small-size geometry or a single combination of influencing factors, and the general flow and heat transfer characteristics are not systematically investigated. In addition, another important factor-the non-Newtonian behavior of the crude oil-is not taken into consideration. In this paper, the convection heat transfer under different combinations of dimensionless numbers as well as the flow-behavior index $n$ for characterizing the non-Newtonian behavior will be investigated.

\section{Physical and Mathematical Model}

\subsection{Physical Model}

The full physical model of a real tanker should be a three-dimensional one, as shown in Figure 1 (top). However, the calculation of a 3D model is too much time-consuming, since the geometry of the oil tanker is usually very large. Considering the longitude temperature gradient is very weak compared to the transverse one, the physical model can be simplified to a two-dimensional one shown in Figure 1 (bottom), in which the red part is crude oil, the green part is ballast water, and the blue part is air (inert gas). 
For the convenience of establishment and computation of the mathematical model, the following assumptions were made:

(1) The upper part of the oil tank is a single layer wall without any building above, which directly touches the air;

(2) The oil tank contains two thirds of the oil; the initial temperature of the oil is $323.15 \mathrm{~K}$;

(3) The four wing spaces at the bottom are filled with ballast water, while the other wing tanks are filled with air. The initial temperature of water and air is $293.15 \mathrm{~K}$;

(4) The tanker's draught is two thirds of its height;

(5) The outside boundary of the tanker is subjected to the third boundary conditions. The temperature of the external sea water and air are both $291.15 \mathrm{~K}$, and the convective heat transfer coefficient between walls and sea water was set to be $1250 \mathrm{~W} /\left(\mathrm{m}^{2} \cdot \mathrm{K}\right)$, and that between walls and air was $50 \mathrm{~W} /\left(\mathrm{m}^{2} \cdot \mathrm{K}\right)$, respectively [16].

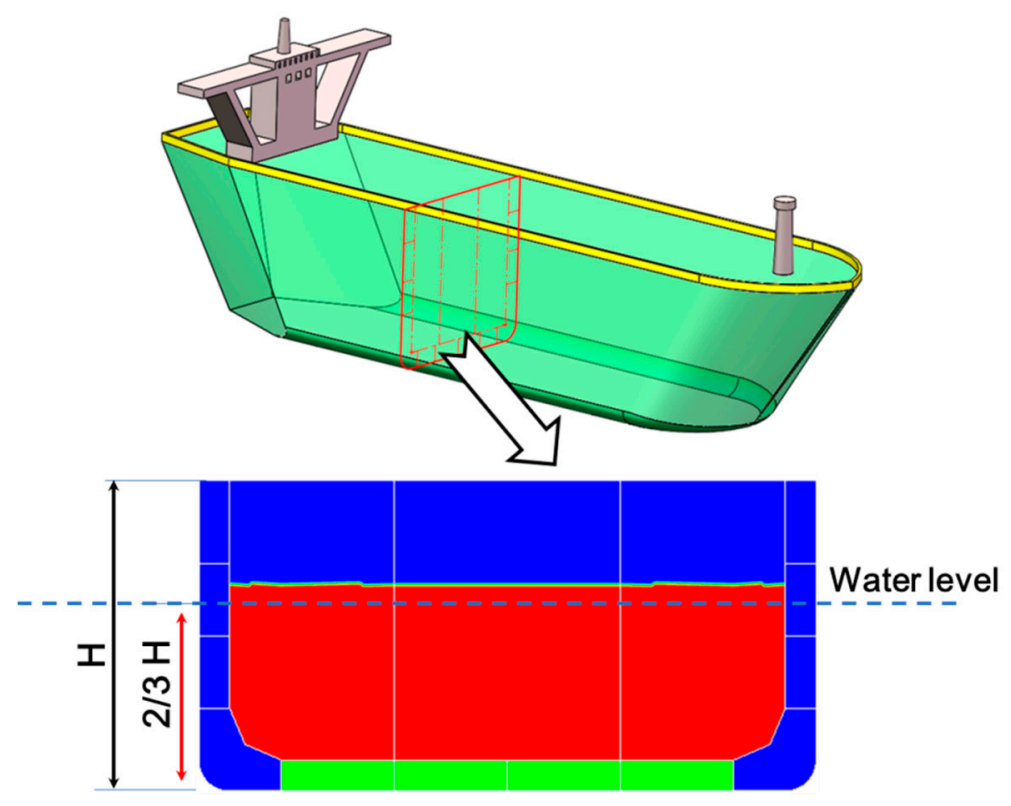

Figure 1. Physical model of the thermal system of an oil tanker.

\subsection{Mathematical Model}

The mathematical model was established in a non-inertial coordinate system shown in Figure 2. The origin of the coordinates was located at the center of the bottom wall. The xoy coordinate system is a non-inertial coordinate system at rest relative to the model; the $x^{\prime} o y^{\prime}$ coordinate system is a static coordinate system. At $t=0$, the model is stationary, and the model starts to move since $t>0$ with the following parameters.

$$
\left\{\begin{array}{c}
\theta=\theta_{m} \sin \left(\frac{2 \pi}{T_{c}} t\right) \\
\omega=\frac{2 \pi}{T_{c}} \theta_{m} \cos \left(\frac{2 \pi}{T_{c}} t\right) \\
\frac{d \omega}{d t}=-\frac{4 \pi^{2}}{T_{2}^{c}} \theta_{m} \sin \left(\frac{2 \pi}{T_{c}} t\right)
\end{array}\right.
$$

Here, $\theta_{m}$ and $T_{c}$ are the amplitude and period of the rolling motion, respectively.

During the rotation, the crude oil in the tanker is subjected to the inertial force caused by the rotation, including centrifugal force, Euler force, and Coriolis force. All the inertial forces are listed below:

(1) Gravity force, $\rho g$; 
(2) Centrifugal force, $-\rho \omega \times(\omega \times r)$;

(3) Euler force, $-\rho \frac{d \omega}{d t} \times r$;

(4) Coriolis force, $-2 \rho \omega \times u$.

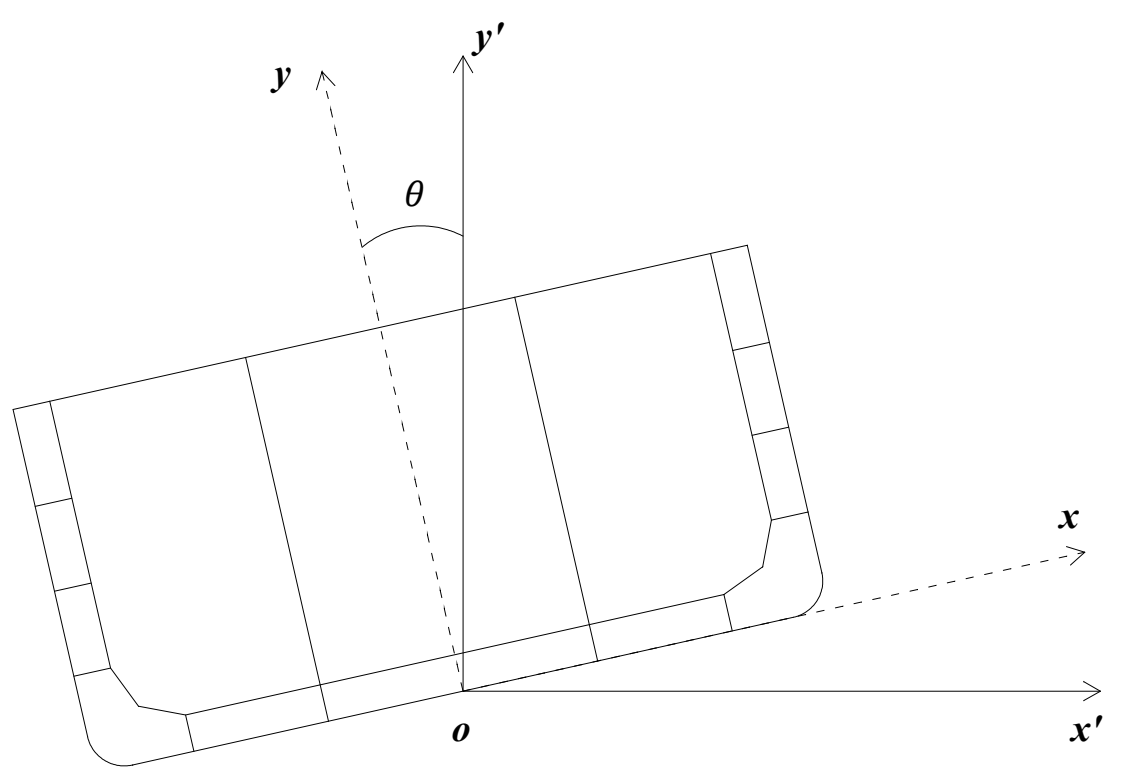

Figure 2. Non-inertial coordinate system.

In the non-inertial coordinate system, the direction of gravity acceleration changes with time, and Rodrigues' rotation formula [17] is used to determine the gradational acceleration in the non-inertial coordinate system, that is

$$
g^{*}=\left(\begin{array}{c}
-g \sin \theta \\
-g \cos \theta \\
0
\end{array}\right) .
$$

With the Boussinesq assumption, the governing equations are given as follows.

The continuous equation:

$$
\frac{\partial u_{x}}{\partial x}+\frac{\partial u_{y}}{\partial y}=0
$$

The momentum equation:

$$
\begin{aligned}
& \frac{\partial\left(\rho_{0} u_{x}\right)}{\partial t}+\frac{\partial\left(\rho_{0} u_{x} u_{x}\right)}{\partial x}+\frac{\partial\left(\rho_{0} u_{x} u_{y}\right)}{\partial y}=-\frac{\partial p_{e f f}}{\partial x}+\frac{\partial}{\partial x}\left(\mu \frac{\partial u_{x}}{\partial x}\right)+\frac{\partial}{\partial y}\left(\mu \frac{\partial u_{x}}{\partial y}\right) \\
& +\rho_{0} \beta\left(T-T_{0}\right) g \sin \theta-\rho_{0} \beta\left(T-T_{0}\right) \omega^{2} x+\rho_{0} \frac{d \omega}{d t} y+2 \rho_{0} \omega u_{y} \\
& \frac{\partial\left(\rho_{0} u_{y}\right)}{\partial t}+\frac{\partial\left(\rho_{0} u_{y} u_{x}\right)}{\partial x}+\frac{\partial\left(\rho_{0} u_{y} u_{y}\right)}{\partial y}=-\frac{\partial p_{e f f}}{\partial y}+\frac{\partial}{\partial x}\left(\mu \frac{\partial u_{y}}{\partial x}\right)+\frac{\partial}{\partial y}\left(\mu \frac{\partial u_{y}}{\partial y}\right) \\
& +\rho_{0} \beta\left(T-T_{0}\right) g \cos \theta-\rho_{0} \beta\left(T-T_{0}\right) \omega^{2} y-\rho_{0} \frac{d \omega}{d t} x-2 \rho_{0} \omega u_{x}
\end{aligned}
$$

From Equations (3)-(5), $u_{x}$ and $u_{y}$ are velocities in $x$ and $y$ direction, respectively; $\rho_{0}$ is the reference density of the crude oil, and the subscript " 0 " here indicates the reference value; $\beta$ is the thermal expansion coefficient; $\omega$ is the rotation angular velocity. $p_{\text {eff }}$ is the effective pressure defined as:

$$
\begin{gathered}
-\frac{\partial p_{e f f}}{\partial x}=-\frac{\partial p}{\partial x}-\rho_{0} g \sin \theta+\rho_{0} \omega^{2} x \\
-\frac{\partial p_{e f f}}{\partial y}=-\frac{\partial p}{\partial y}-\rho_{0} g \cos \theta+\rho_{0} \omega^{2} y
\end{gathered}
$$


The energy equation:

$$
\frac{\partial\left(\rho_{0} T\right)}{\partial t}+\frac{\partial\left(\rho_{0} u_{x} T\right)}{\partial x}+\frac{\partial\left(\rho_{0} u_{y} T\right)}{\partial y}=\frac{\partial}{\partial x}\left(\frac{\lambda}{c_{p}} \frac{\partial T}{\partial x}\right)+\frac{\partial}{\partial y}\left(\frac{\lambda}{c_{p}} \frac{\partial T}{\partial y}\right) .
$$

Here, $\lambda$ and $c_{p}$ are conductivity and specific heat capacity of the crude oil.

Gas-liquid phase distribution function:

$$
\frac{\partial \alpha_{q}}{\partial t}+\frac{\partial\left(u_{x} \alpha_{q}\right)}{\partial x}+\frac{\partial\left(u_{y} \alpha_{q}\right)}{\partial y}=0 .
$$

Here, $\alpha_{q}$ is the volume fraction of the $q$ th phase.

Surface tension is considered in the calculation, based on the continuum surface force model (CSF) proposed by Brackbill et al. [18]; surface tension is added as a source term to the momentum equation

$$
F_{c s f}=\sum_{i<j} \sigma_{i j} \frac{\alpha_{i} \rho_{i} k_{j} \nabla a_{j}+\alpha_{j} \rho_{j} k_{i} \nabla a_{i}}{\frac{1}{2}\left(\rho_{i}+\rho_{j}\right)} .
$$

In this model, because there are only two phases, air and crude oil, in a cell, the equation can be simplified as follows.

$$
F_{c s f}=\sigma_{12} \frac{\rho k_{1} \nabla a_{1}}{\frac{1}{2}\left(\rho_{1}+\rho_{2}\right)}
$$

Here, $\rho$ is the volume averaged density and determined by $\rho=\alpha_{1} \rho_{1}+\left(1-\alpha_{1}\right) \rho_{2}$.

In addition, the $\mathrm{Nu}$ number used in this paper is calculated by the following formulas [19].

$$
N u_{X}=-\frac{\partial \Theta}{\partial X}, N u_{Y}=-\frac{\partial \Theta}{\partial Y}, \overline{N u_{X}}=\int_{\text {min }}^{\max } N u_{X} d X, \overline{N u_{Y}}=\int_{\text {min }}^{\max } N u_{Y} d Y
$$

Governing Equations (3)-(7) are transformed into dimensionless form using the following formulas, and the dimensionless equations are given by Equations (13)-(17).

$$
\begin{aligned}
& X=\frac{x}{l}, Y=\frac{y}{l}, U=\frac{u_{x}}{\omega l}, V=\frac{u_{y}}{\omega l}, \Theta=\frac{T-T_{0}}{T_{h}-T_{0}}, P=\frac{p_{e f f}}{\rho \bar{\omega}^{2} l^{2}} \\
& \tau=\bar{\omega} t, \omega^{*}=\frac{\bar{\omega} l^{2}}{\alpha}, \mu^{*}=\frac{\mu}{\mu_{0}}, \operatorname{Pr}=\frac{v_{0}}{\alpha}, R a=\frac{g \beta \Delta T l^{3}}{v_{0} \alpha}, R a_{w}=\frac{\bar{\omega} l \beta \Delta T l^{3}}{v_{0} \alpha} \\
& R e=\frac{\bar{\omega} l^{2}}{v}, R i=\frac{G r}{R e^{2}}=\frac{1}{\omega^{* 2}} \operatorname{Pr} R a
\end{aligned}
$$

In Equation (11), $R a_{w}$ is the rotational Rayleigh number, $\omega^{*}$ is the rotation-strength number characterizing the importance of centrifugal force relative to diffusivity, $R i$ is the Richardson number, $l$ is the reference length which equates the width of the largest tank, and $\bar{\omega}$ is the average angular velocity calculated by Equation (12).

$$
\bar{\omega}=\frac{2 \int_{-\frac{T}{4}}^{\frac{T}{4}} \omega d t}{T_{\mathcal{C}}}=\frac{4 \theta_{m}}{T_{\mathcal{C}}}
$$

Dimensionless governing equations are written as follows.

$$
\frac{\partial U}{\partial X}+\frac{\partial V}{\partial Y}=0
$$




$$
\begin{gathered}
\frac{\partial U}{\partial \tau}+\frac{\partial(U U)}{\partial X}+\frac{\partial(U V)}{\partial Y}=-\frac{\partial P}{\partial X}+\frac{P r}{\omega^{*}} \frac{\partial}{\partial X}\left(\mu^{*} \frac{\partial U}{\partial X}\right)+\frac{P r}{\omega^{*}} \frac{\partial}{\partial Y}\left(\mu^{*} \frac{\partial V}{\partial Y}\right) \\
+R i \Theta \sin \left(\theta_{m} \sin \tau\right)-R i \Theta g^{*} X \theta_{m}^{2} \cos ^{2} \tau-\theta_{m} Y \sin \tau+2 \theta_{m} \cos \tau V \\
\frac{\partial V}{\partial \tau}+\frac{\partial(U V)}{\partial X}+\frac{\partial(V V)}{\partial Y}=-\frac{\partial P}{\partial Y}+\frac{P r}{\omega^{*}} \frac{\partial}{\partial X}\left(\mu^{*} \frac{\partial V}{\partial X}\right)+\frac{P r}{\omega^{*}} \frac{\partial}{\partial Y}\left(\mu^{*} \frac{\partial V}{\partial Y}\right) \\
+R i \Theta \cos \left(\theta_{m} \sin \tau\right)+R i \Theta g^{*} Y \theta_{m}^{2} \cos ^{2} \tau+\theta_{m} X \sin \tau-2 \theta_{m} \cos \tau U \\
\frac{\partial(\Theta)}{\partial \tau}+\frac{\partial(U \Theta)}{\partial X}+\frac{\partial(V \Theta)}{\partial Y}=\frac{1}{\omega^{*}}\left(\frac{\partial^{2} \Theta}{\partial X^{2}}+\frac{\partial^{2} \Theta}{\partial Y^{2}}\right) \\
\frac{\partial \alpha_{q}}{\partial \tau}+\frac{\partial\left(U \alpha_{q}\right)}{\partial X}+\frac{\partial\left(V \alpha_{q}\right)}{\partial Y}=0
\end{gathered}
$$

In Equations (14) and (15), $g^{*}$ is the dimensionless centrifugal acceleration and is determined by $g^{*}=\bar{\omega}^{2} l / g$.

It can be found from Equations (13)-(17) that there are four independent dimensionless parameters, $\operatorname{Pr}, \omega^{*}, R i$, and $\theta_{m}$. In this research, the properties and amplitude of rolling are fixed, so $\omega^{*}$ and $R i$ are the independent variables that regulate the flow and heat transfer behavior. Besides, in the non-Newtonian behavior cases, which will be introduced in detail below, flow-behavior index $n, \omega^{*}$, and $R i$ are the three independent dimensionless variables.

Boundary conditions:

$$
\begin{aligned}
& L / 2<x<L / 2, y=H \quad: \quad T=293.15 \mathrm{~K}, h=50 \mathrm{~W} /\left(\mathrm{m}^{2} \cdot \mathrm{K}\right) \\
& x=-L / 2,0<y<2 L / 3 \quad: \quad T=291.15 \mathrm{~K}, h=1250 \mathrm{~W} /\left(\mathrm{m}^{2} \cdot \mathrm{K}\right) \\
& x=-L / 2,2 L / 3<y<L \quad: \quad T=293.15 \mathrm{~K}, h=50 \mathrm{~W} /\left(\mathrm{m}^{2} \cdot \mathrm{K}\right) \\
& x=L / 2,0<y<2 L / 3 \quad: \quad T=291.15 \mathrm{~K}, h=1250 \mathrm{~W} /\left(\mathrm{m}^{2} \cdot \mathrm{K}\right) \\
& x=L / 2,2 L / 3<y<L \quad: \quad T=293.15 \mathrm{~K}, h=50 \mathrm{~W} /\left(\mathrm{m}^{2} \cdot \mathrm{K}\right) \\
& -L / 2<x<L / 2, y=0 \quad: \quad T=291.15 \mathrm{~K}, h=1250 \mathrm{~W} /\left(\mathrm{m}^{2} \cdot \mathrm{K}\right)
\end{aligned}
$$

For easy reference, boundary conditions are also illustrated in Figure 3.

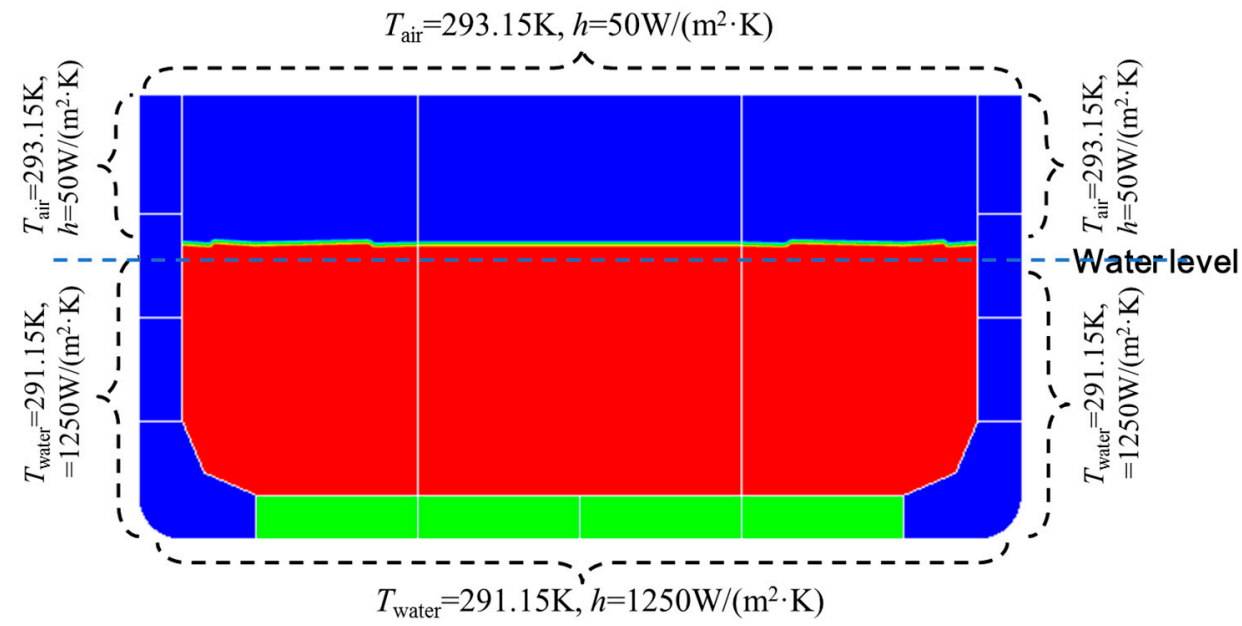

Figure 3. Boundary conditions of the mathematical model.

\section{Numerical Methods and Their Validation}

\subsection{Numerical Methods}

The basic equations for the present three-dimensional unsteady rotating cavity flow were solved numerically in the framework of the control-volume method by Ansys-Fluent 17.0 solver. In particular, 
the power-law scheme was used to discretize Equations (3)-(7) on a non-staggered grid system with the pressure and temperature defined at the mesh centers. The convection terms were discretized by a second-order upstream scheme, and the diffusion terms were discretized by the central differencing scheme. The resulting finite difference equations were solved by the SIMPLE algorithm [20] with a $k-e$ model for turbulence effect. To resolve the steep velocity and temperature gradients in the near-wall region, a non-uniform grid shown in Figure 4 was used.

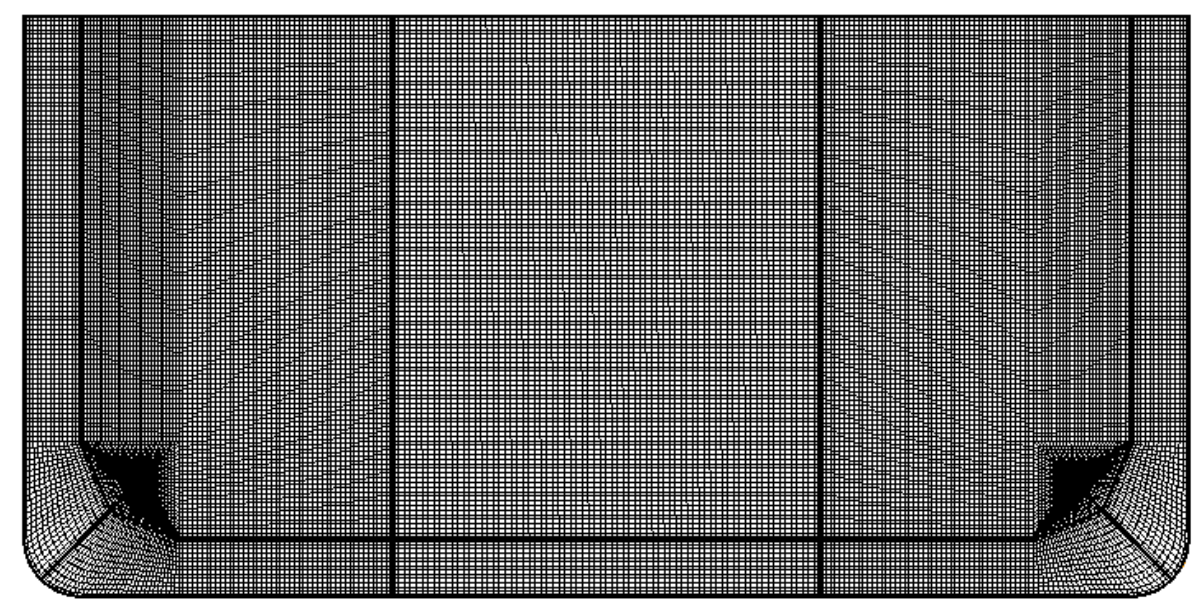

Figure 4. The structural grids generated by ICEM-CFD (Integrated Computer Engineering and Manufacturing code for Computational Fluid Dynamics).

\subsection{Grid-Independence Test}

A grid-independence test was carried out for a rolling case with $R i=144.5$ and $\omega^{*}=4.86 \times 10^{5}$, on three different grids-16,000 cells, 32,000 cells, and 64,000 cells-and the average temperatures are compared in Figure 5. It was found that the results calculated from the grid of 32,000 cells were very close to that calculated from the grid of 64,000 cells. Thus, all the calculations in this paper were performed on the grid of 32,000 cells.

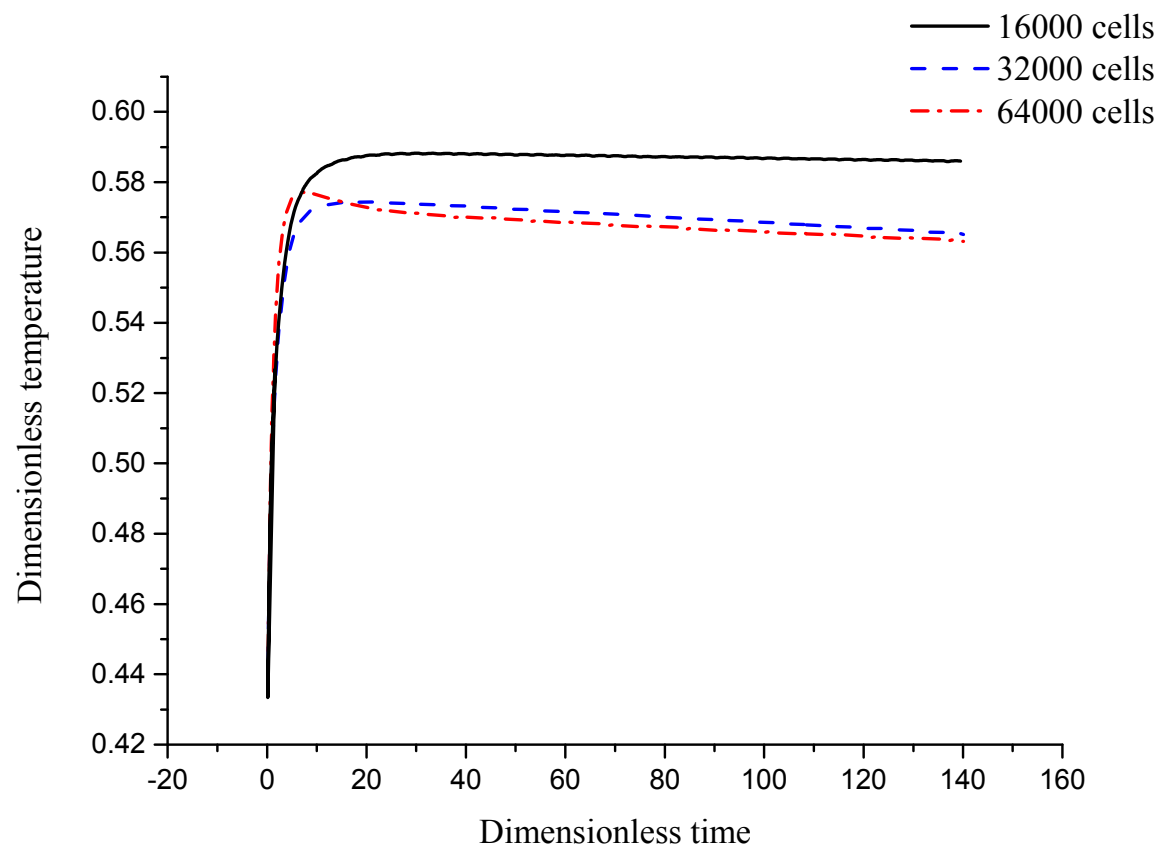

Figure 5. Average temperature calculated on different grid systems. 


\subsection{Model Validation}

The numerical validations were performed with two cases-a partially-filled cavity subjected to a linear acceleration motion for validating the phase interface, and a constant-velocity rotational natural convection case for validation of the heat transfer and flow.

In the first validation case, a partially-filled cavity was accelerated at $3 \mathrm{~m} / \mathrm{s}^{2}$ on a horizontal flat surface. The free surface of the liquid can be determined by an analytical correlation given by Equation (19). The two-phase flow problem was solved by the proposed model at the same time and compared with the analytical correlation, which is shown in Figure 6. In the figure, the black dashed line indicates the analytical results of the free surface, while the contour shows the distribution of the liquid and gas phase. It can be found that the numerical results agree well with the analytical results.

$$
\mathrm{y}=\arctan \frac{3}{9.8} x+0.5=0.3 x+0.5
$$

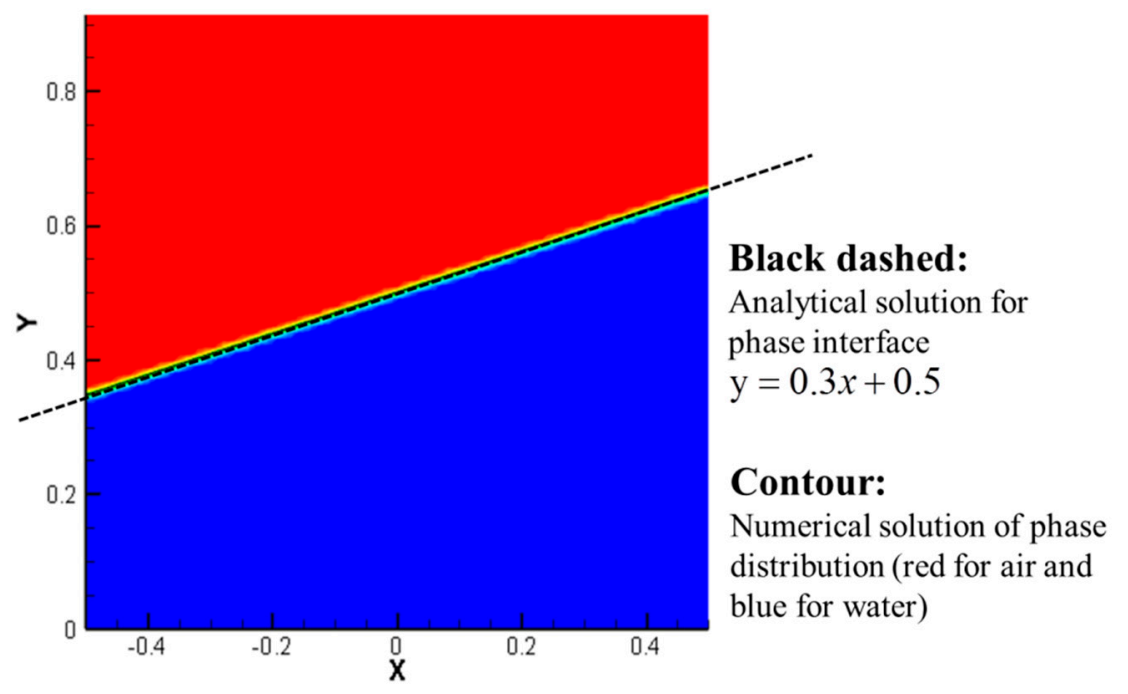

Figure 6. Comparison of phase interface between numerical results and analytical results.

In addition, a natural convection in a rotation system with respect to a horizontal axis, which was an ideal case for the rolling motion in this research, was employed to validate the flow and heat transfer. The numerical results were compared with those calculated by Tso et al. [21] (middle) and the experimental data performed by Saleh et al. [22] (right), as shown in Figure 7. It was found that the numerical results agree well with the results in the literature. 

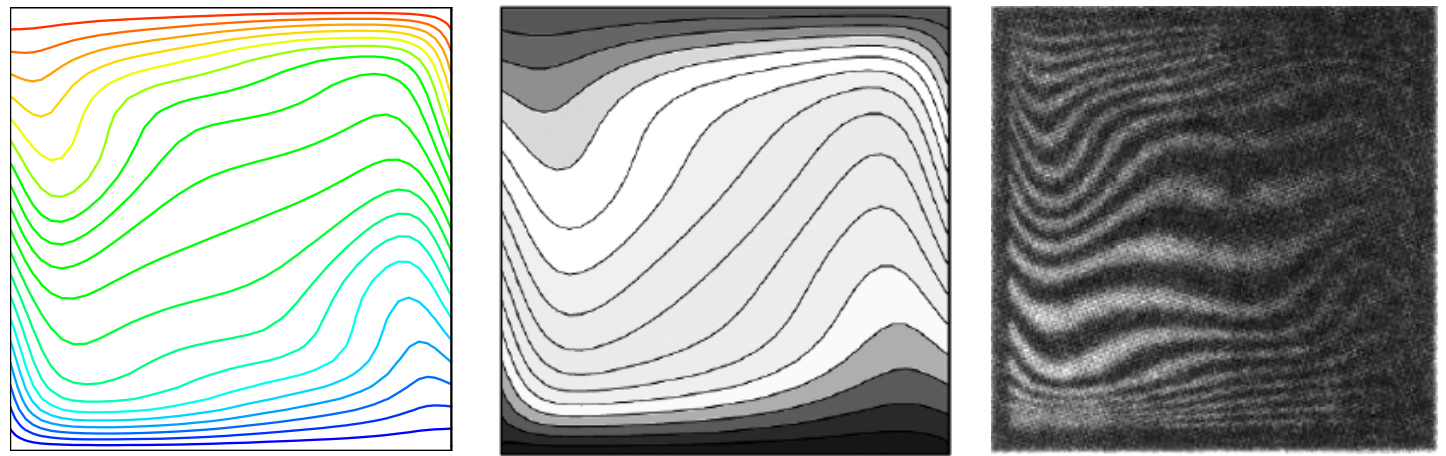

(a) $\phi=0, \mathrm{Ra}=3 \times 10^{5}, \Omega=17.5 \mathrm{rpm}$
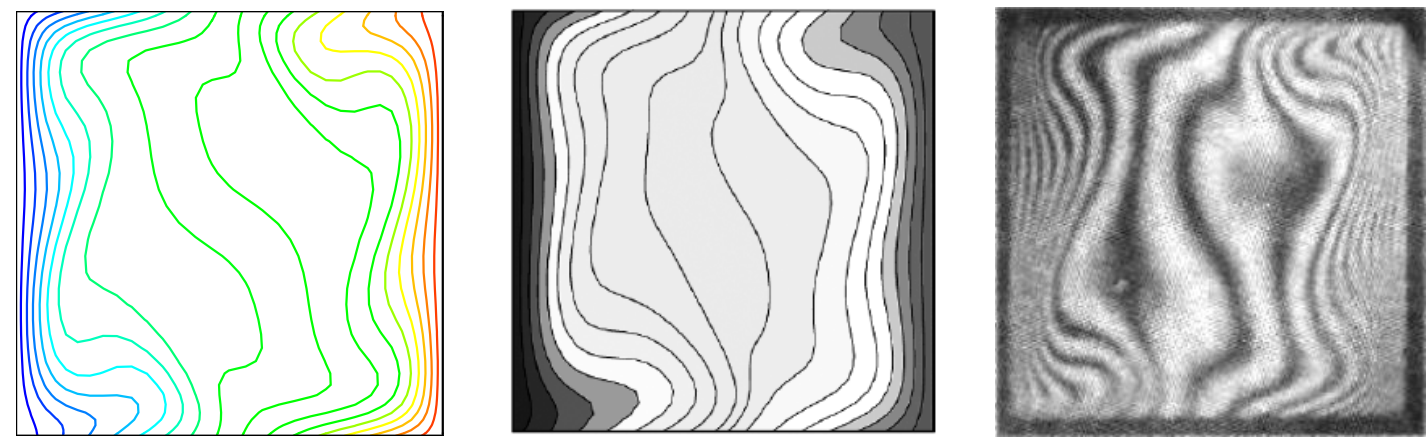

(b) $\phi=3 \pi / 2, \mathrm{Ra}=3 \times 10^{5}, \Omega=15.5 \mathrm{rpm}$
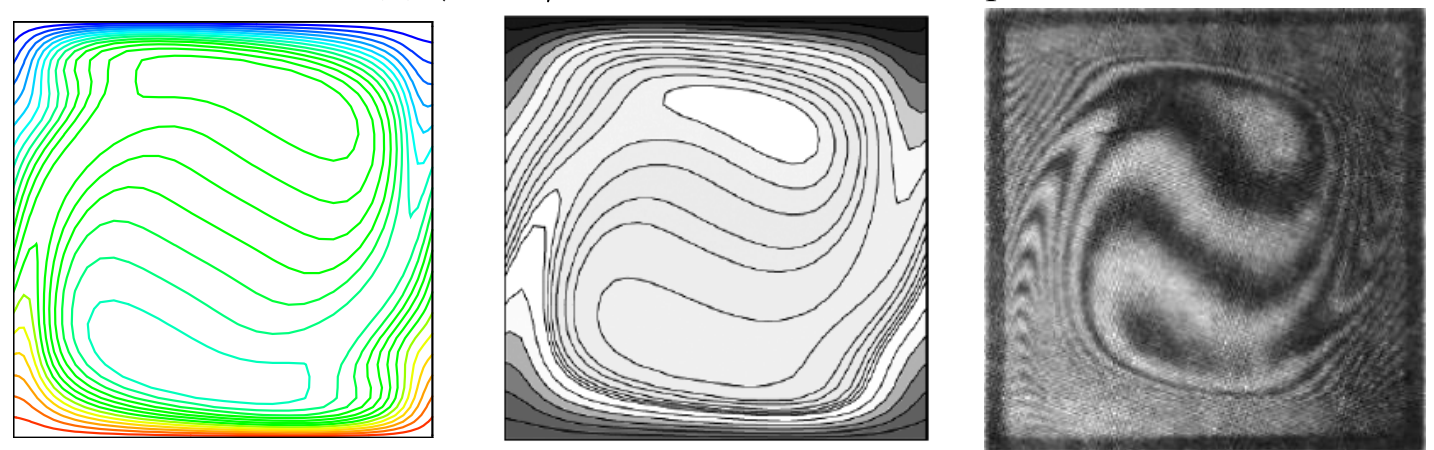

(c) $\phi=\pi, \mathrm{Ra}=3 \times 10^{5}, \Omega=17.5 \mathrm{rpm}$

Figure 7. Comparison of computed isotherms between present work (left) with Tso et al. [21] (middle) and Saleh et al. [22] (right) results under (a) $\phi=0$, Ra $=3 \times 10^{5}, \Omega=17.5 \mathrm{rpm}$, (b) $\phi=3 \pi / 2, \mathrm{Ra}=3 \times 10^{5}, \Omega=15.5 \mathrm{rpm}$, and (c) $\phi=\pi, \mathrm{Ra}=3 \times 10^{5}, \Omega=17.5 \mathrm{rpm}$.

\section{Results and Discussion}

It was discussed in Section 2.2 that $P r, \omega^{*}, R i$, and $\theta_{m}$ are four dimensionless parameters regulating the flow and heat transfer behavior. In this research, the properties and amplitude (10 degrees) of rolling were fixed (a medium value with the normal range [4] was selected), so $\omega^{*}$ and $R i$ were the only two dimensionless independent variables.

When the tanker was subjected to a rolling motion, the surface of the crude oil oscillated accordingly, as shown in Figure 8. Thus, the mechanism of the heat transfer in this research was a mixed convection heat transfer, which can be described by the Richardson number $(R i)$ - the forced convection dominates the heat transfer when $R i<0.1$, natural convection dominates when $R i>10$, and both dominate when $0.1<R i<10$. Thus, the selection of $R i$ ensures the coverage of different ranges. The selection of rotation-strength number $\omega^{*}$ considers the popular angular velocity and tank size in real practice. In this section, 12 cases were designed to investigate effects of different dimensionless parameters as well as non-Newtonian behavior in terms of the flow-behavior index on flow and heat transfer. 
The combinations of the dimensionless parameters for case 1 to 12 are listed in Table 1, in which cases 7-12 consider the non-Newtonian behavior, while cases 1-6 do not consider this behavior. In this research, non-Newtonian behavior is described by a power law with a flow-behavior index $n$; different $n$ indicates different non-Newtonian behavior. It should be noted that case 1 was the reference case that was not subjected to a rolling motion. The designed cases were calculated with the combinations of the dimensionless parameters listed in Table 1.

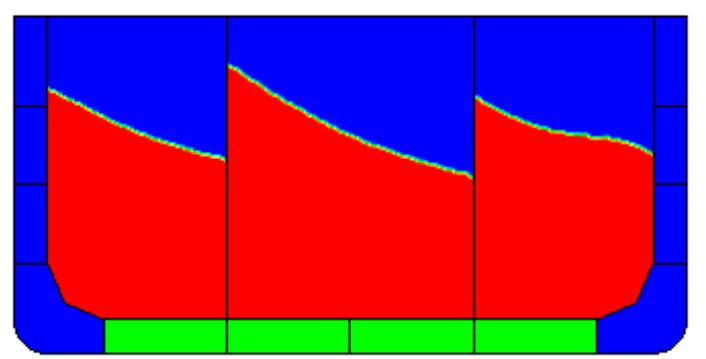

(a)

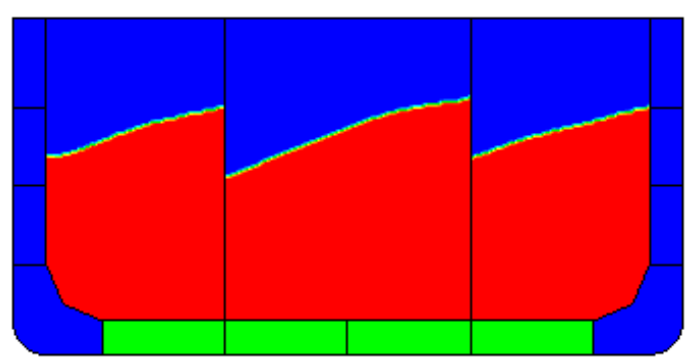

(b)

Figure 8. The gas-liquid phase distribution images at two time instants in case 2: (a) $\tau=27,(\mathbf{b}) \tau=54$.

Table 1. The combinations of the dimensionless parameters for cases 1 to 12 .

\begin{tabular}{cccc}
\hline Case & $\boldsymbol{R i}$ & $\boldsymbol{\omega}^{*}$ & $\boldsymbol{n}$ \\
\hline Case 1 & $\infty$ & 0 & 1 \\
Case 2 & 1 & $1.67 \times 10^{8}$ & 1 \\
Case 3 & 5 & $1.67 \times 10^{8}$ & 1 \\
Case 4 & 10 & $1.67 \times 10^{8}$ & 1 \\
Case 5 & 5 & $1.67 \times 10^{7}$ & 1 \\
Case 6 & 5 & $1.67 \times 10^{6}$ & 1 \\
Case 7 & 5 & $1.67 \times 10^{8}$ & 0.5 \\
Case 8 & 5 & $1.67 \times 10^{8}$ & 0.7 \\
Case 9 & 5 & $1.67 \times 10^{8}$ & 0.9 \\
Case 10 & $\infty$ & 0 & 0.5 \\
Case 11 & $\infty$ & 0 & 0.7 \\
Case 12 & $\infty$ & 0 & 0.9 \\
\hline
\end{tabular}

\subsection{The Effect of Ri with Fixed $\omega^{*}$}

As discussed at the beginning of Section 4, the Richardson number Ri represents the importance of the natural convection relative to the forced convection, and $\omega^{*}$ characterizes the importance of centrifugal forces due to the rolling motion, relative to diffusivity. It can be concluded that $R i$ and $\omega^{*}$ have effects on the thermal-hydraulic behavior of the crude oil. In this part, three different $R i$, lying in different ranges of mixed convection, were investigated with fixed $\omega^{*}$ in cases 2 to 4 . The results were compared with each other and with those of the reference case (case 1).

First, the temperature and the velocity fields at $\tau=11,500$ are compared in Figure 9. It can be found that the temperature field presents a forced convection dominated distribution when $R i$ was low. When $R i$ increases, the flow and heat transfer was gradually dominated by natural convection. Figure 10 compares the dimensionless temperature drop of different $R i$. Together with Figure 9, it was found that the temperature drops faster at lower $R i$, since forced convection enhances the heat exchange. 

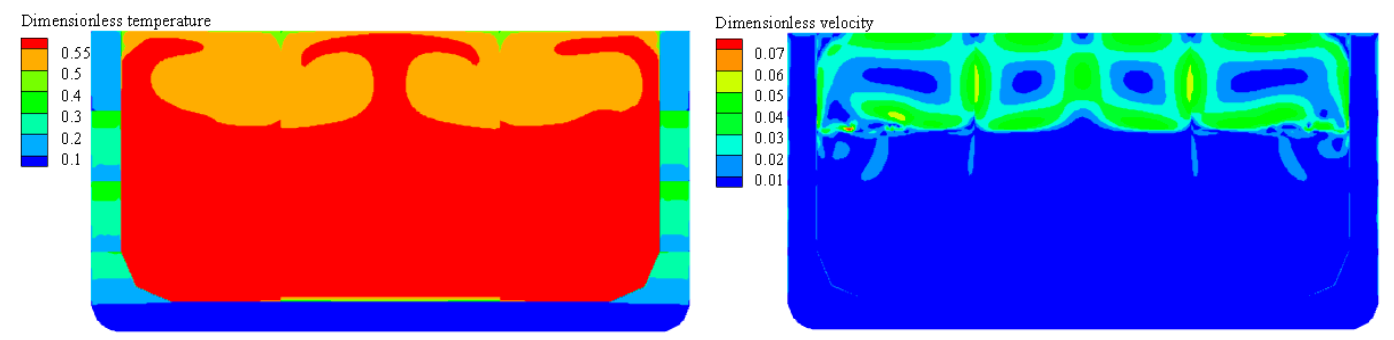

(a) Case 1: $R i=\infty$
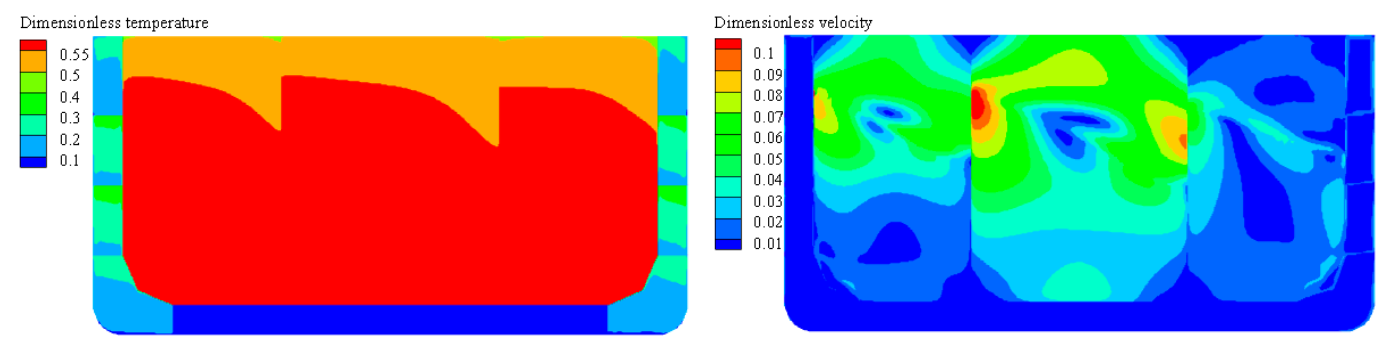

(b) Case 2: $R i=1$
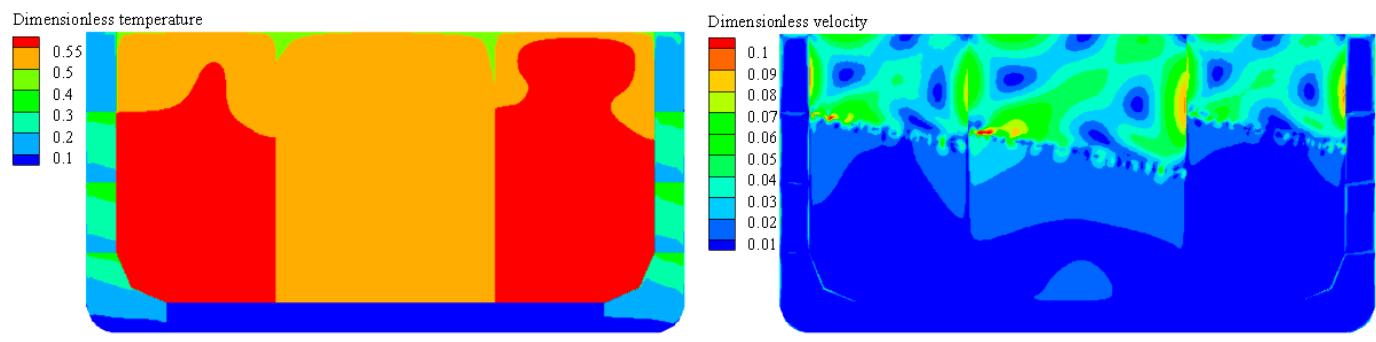

(c) Case 3: $R i=5$
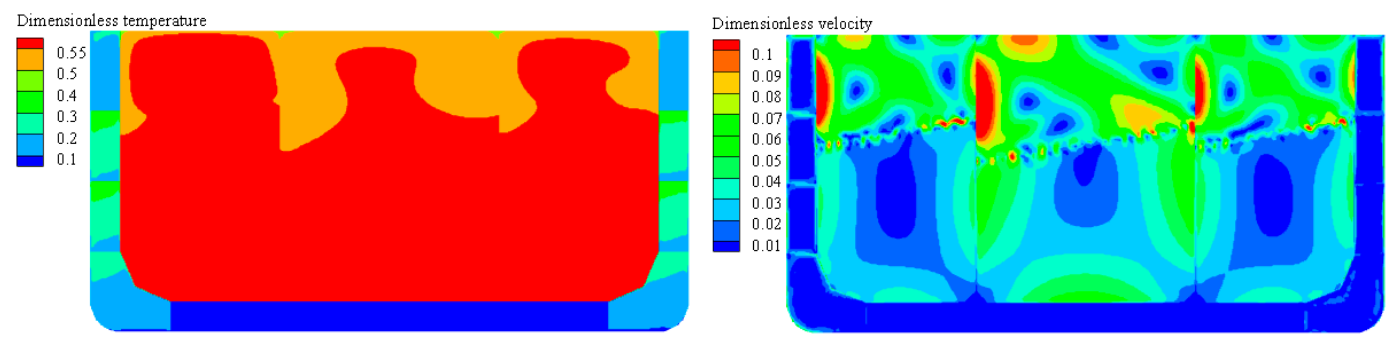

(d) Case 4: $R i=10$

Figure 9. The temperature and the velocity fields for cases 1-4 at $\tau=11,500$ : (a) Case 1, (b) Case 2, (c) Case 3, (d) Case 4. 


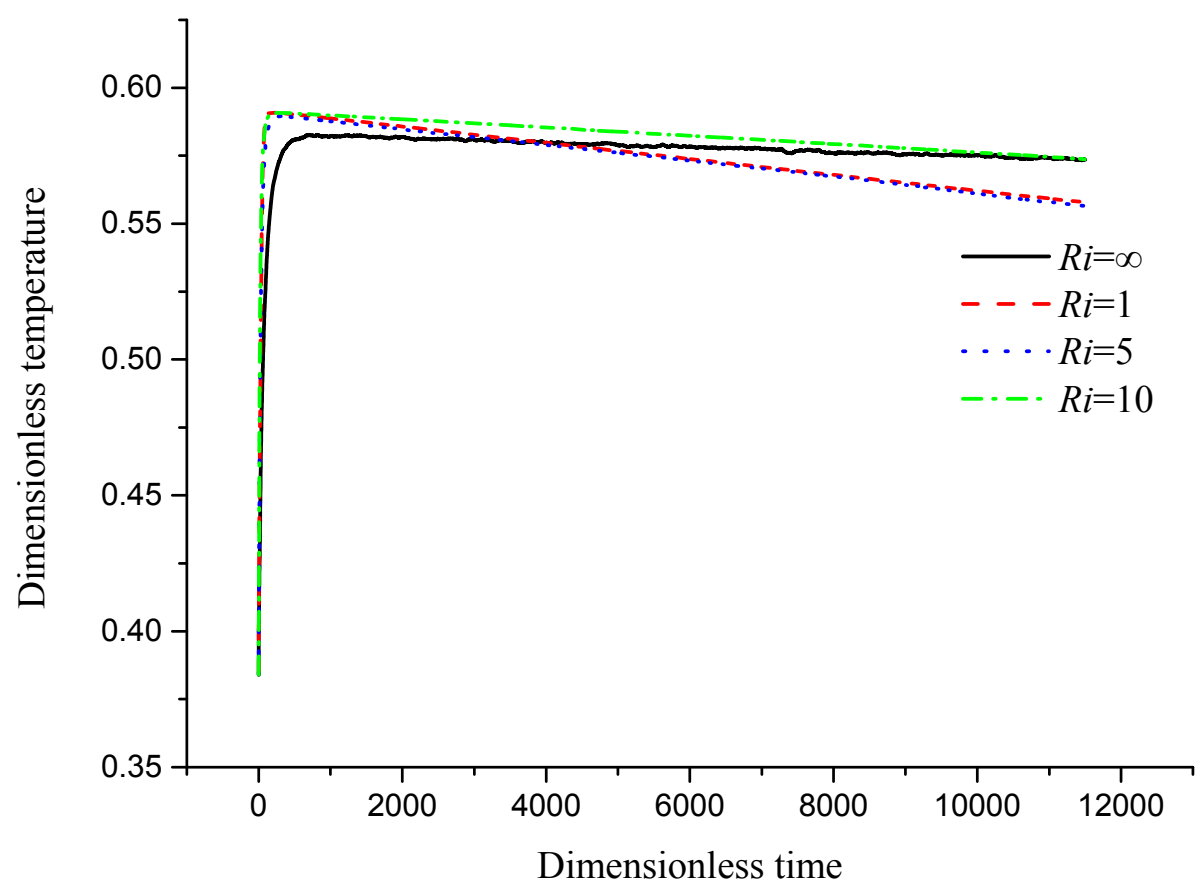

Figure 10. Comparison of temperature drop among cases 1 to 4 .

For further analysis of the effect of rolling on the thermal process, the cycle-averaged Nusselt numbers on some typical boundaries shown in Figure 11 at five different time instants were compared among cases 1 to 4 in Figure 12. It was found that the largest Nusselt number corresponded to the lowest $R i$, and the smallest Nusselt number corresponded to the largest $R i$. However, for moderate $R i$, such an obvious characteristic was not found, since the influence of angle effect on natural convection plays an inevitable role.

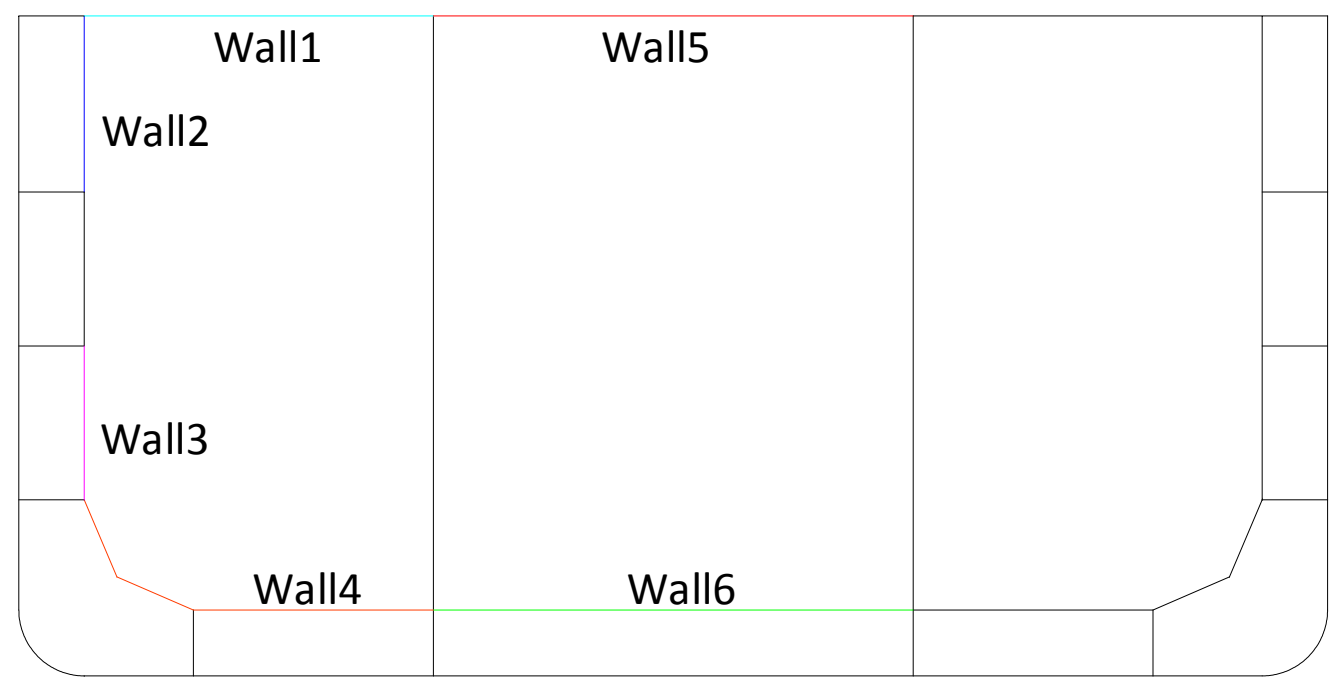

Figure 11. Walls under observation for Nusselt numbers. 


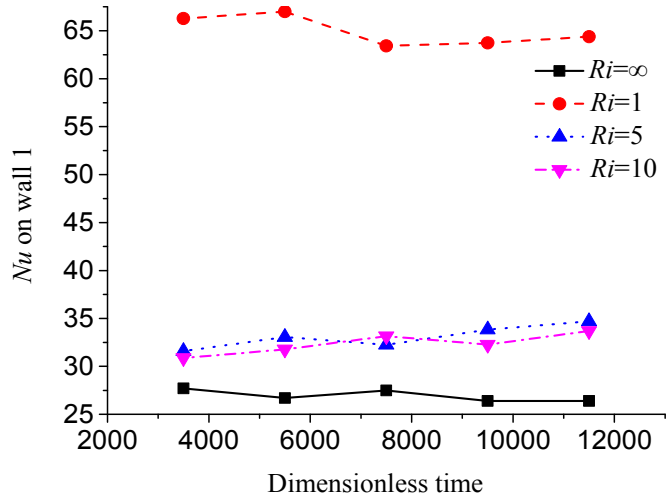

(a)

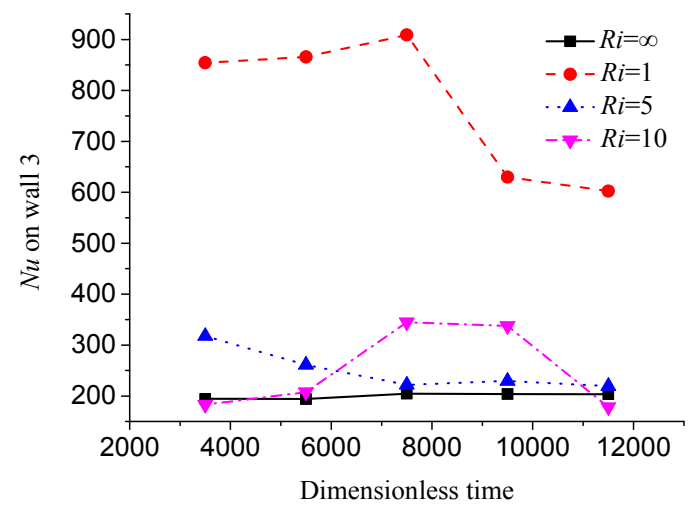

(c)

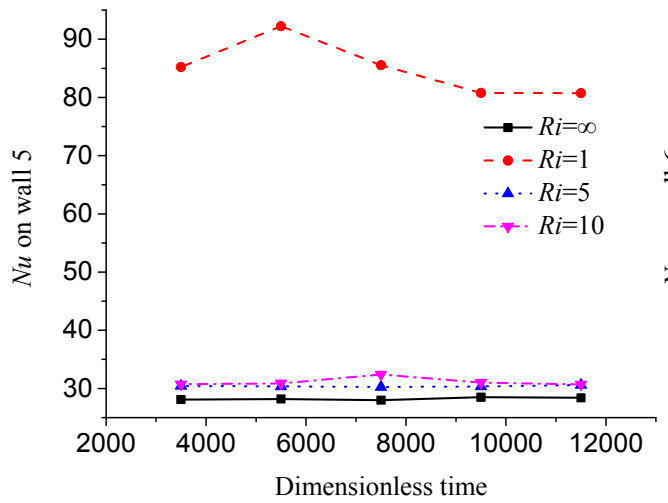

(e)

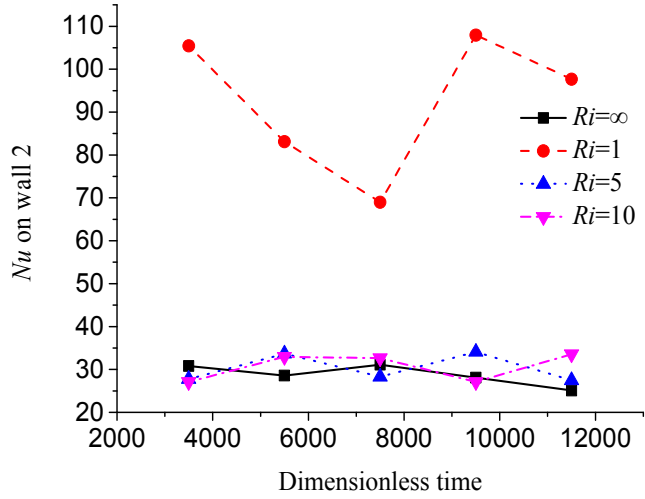

(b)

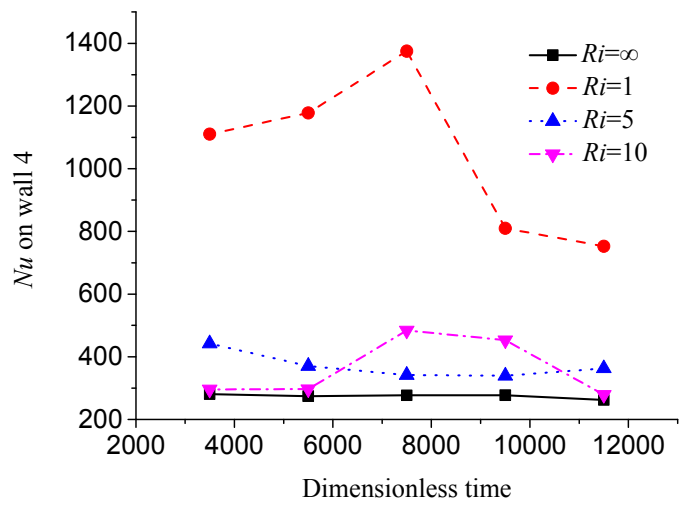

(d)

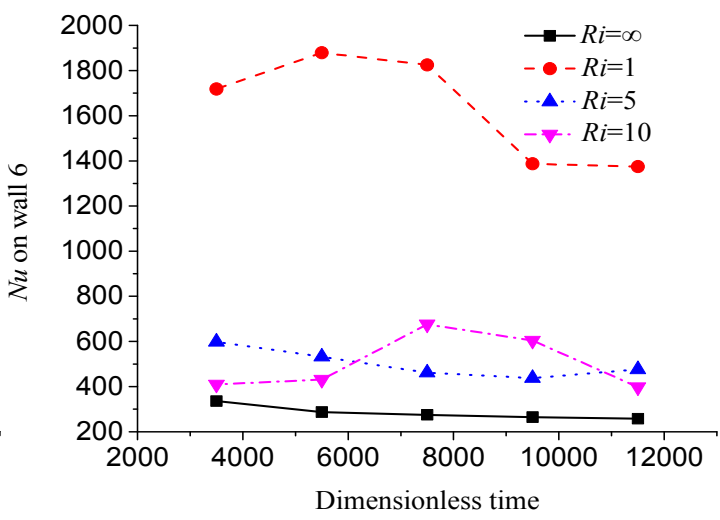

(f)

Figure 12. The $N u$ number on different walls in cases 1, 2, 3, and 4: (a) $N u$ on wall 1, (b) $N u$ on wall 2, (c) $N u$ on wall 3, (d) Nu on wall 4, (e) $N u$ on wall 5, (f) $N u$ on wall 6.

\subsection{The Effect of $\omega^{*}$ with Fixed Ri}

For the rolling motion encountered by the oil tanker, the rotational angular velocity is small and usually changes in a narrow range. Therefore, large variation in $\omega^{*}$ is usually caused by the change of geometry size. Therefore, $\omega^{*}$ is dominated by the geometry size, which is different from a small-size model. In this part, three different $\omega^{*}$ were investigated with fixed $R i$ in cases 3,5 , and 6 . The results were compared with each other, and some instantaneous temperature and velocity fields are shown in Figure 13. It was shown that the temperature drops faster at lower $\omega^{*}$, since lower $\omega^{*}$ corresponded to 
smaller geometry size within a small angular velocity range, and thus led to a faster temperature drop. Figure 14 further indicates that lower $\omega^{*}$ corresponded to a faster temperature drop.
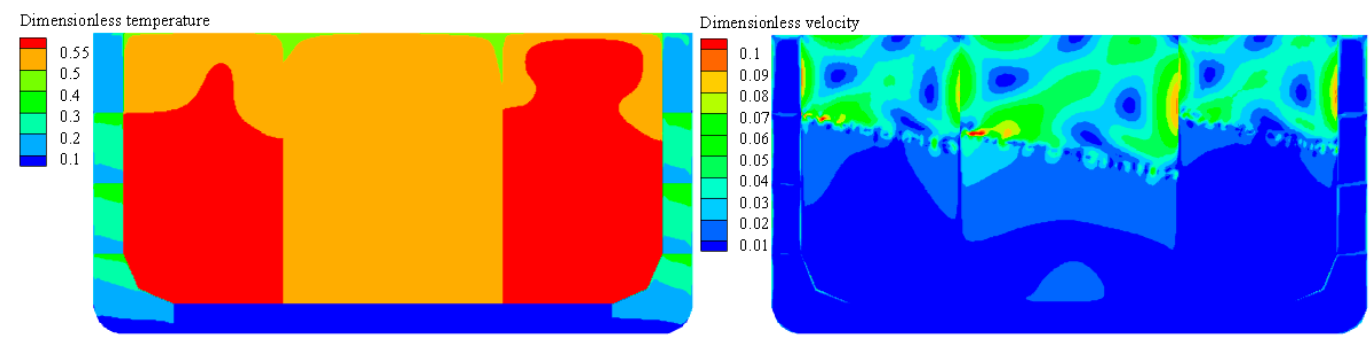

(a) Case 3: $\omega^{*}=1.4 \times 10^{9}$
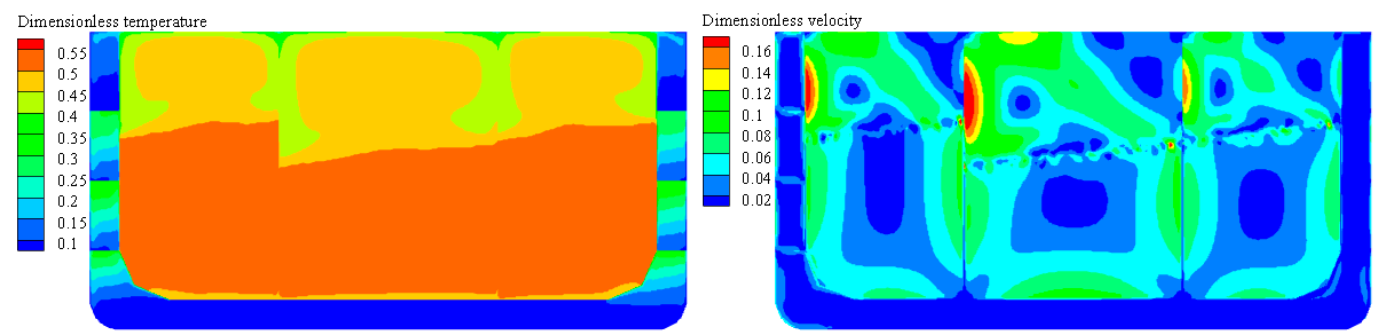

(b) Case 5: $\omega^{*}=1.4 \times 10^{8}$
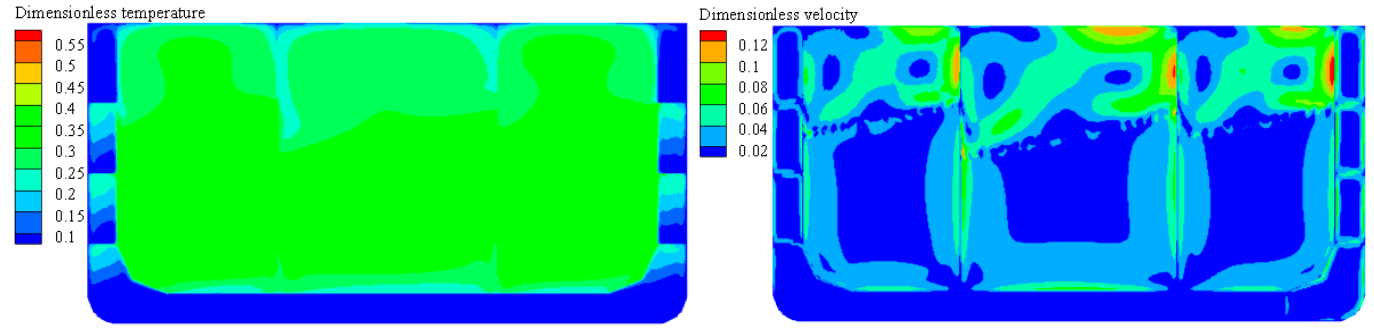

(c) Case 6: $\omega^{*}=1.4 \times 10^{7}$

Figure 13. The temperature field and the velocity field for cases 3, 5, and 6 at $\tau=11,500$ : (a) Case 1 , (b) Case 2, (c) Case 3.

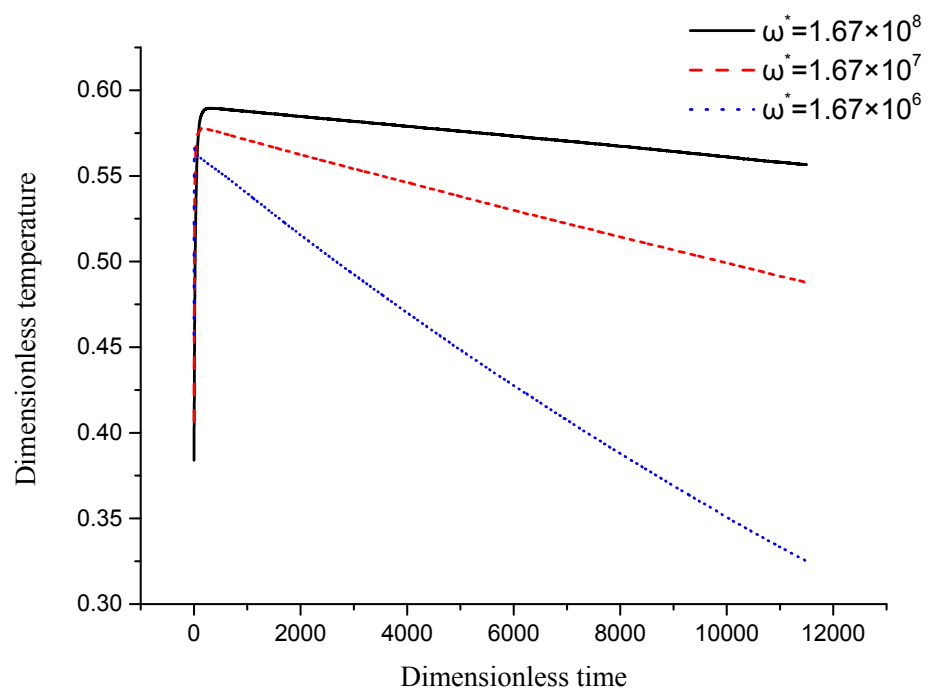

Figure 14. Comparison of temperature drop among cases 3, 5, and 6 . 
The comparisons of boundary Nusselt numbers are shown in Figure 15. It was also found that lower $\omega^{*}$ corresponded to a larger Nusselt number.

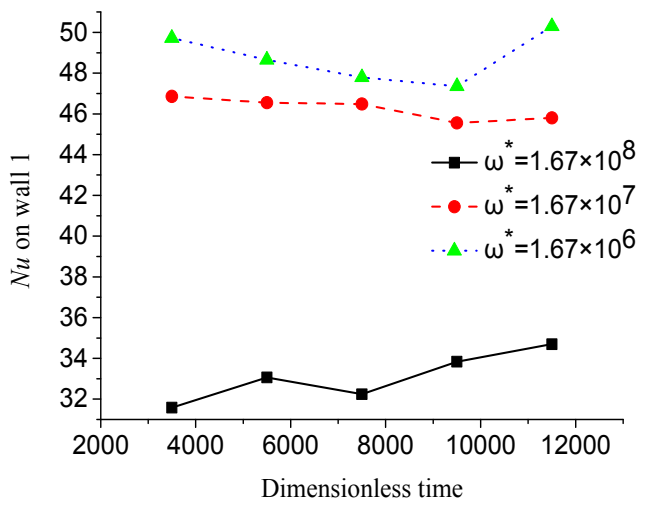

(a)

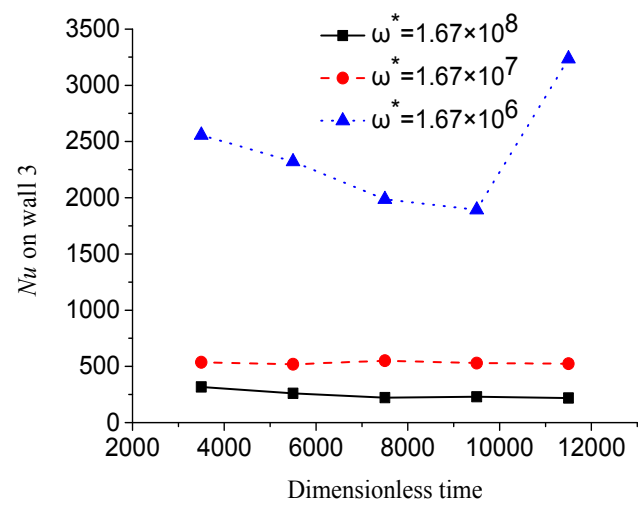

(c)

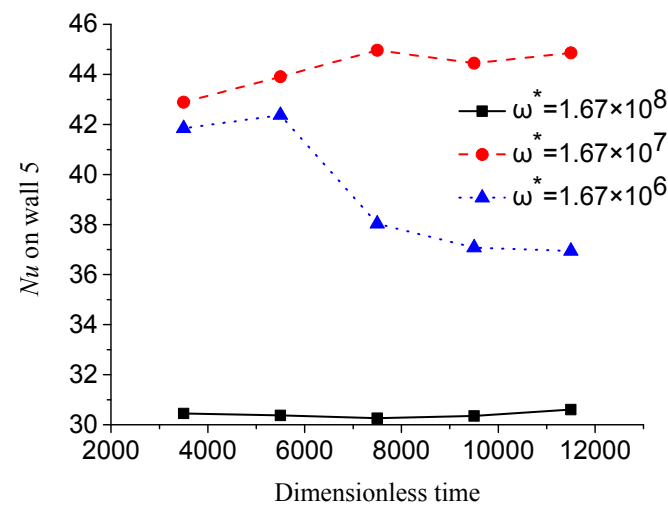

(e)

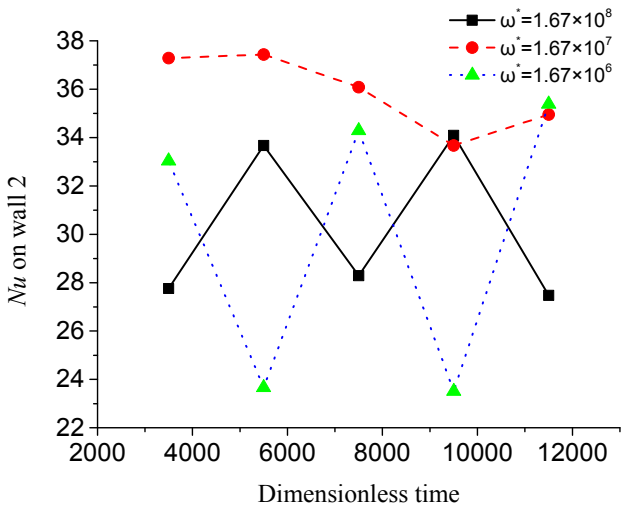

(b)

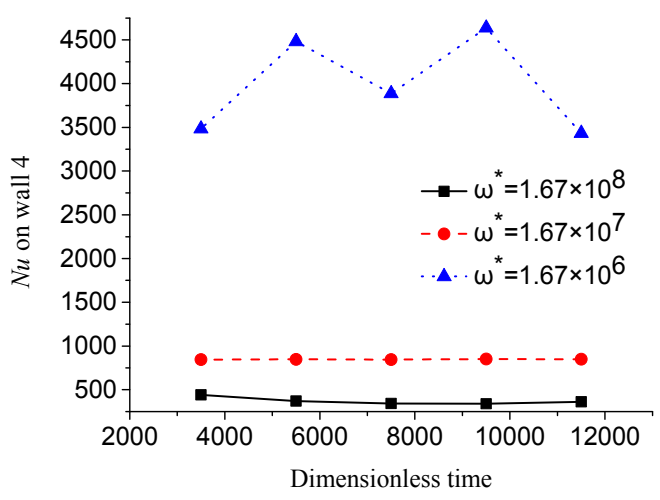

(d)

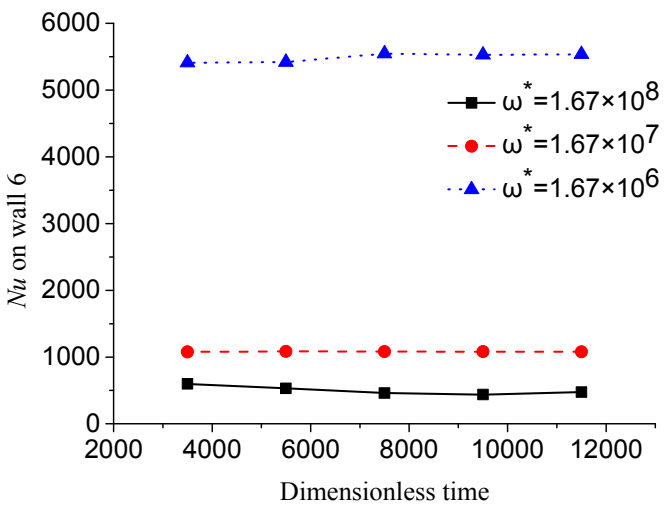

(f)

Figure 15. Comparison of boundary Nusselt numbers between cases 3, 5, and 6: (a) $N u$ on wall 1, (b) $\mathrm{Nu}$ on wall 2, (c) $\mathrm{Nu}$ on wall 3, (d) $\mathrm{Nu}$ on wall 4, (e) $\mathrm{Nu}$ on wall 5, (f) $\mathrm{Nu}$ on wall 6.

\subsection{The Effect of Non-Newtonian Behavior with Fixed $\omega^{*}$ and Ri}

When the fluid was subjected to a rolling motion, the flow velocity was larger, which can be found in Figures 9 and 13. Thus, the shear rate in the rolling case should be large, which influences the viscosity of non-Newtonian fluids. In this part, the effect of different non-Newtonian behavior, represented by the flow-behavior index $n$, was studied for the static case and the rolling case, and the 
influence of non-Newtonian behavior on thermal-hydraulic process was clarified. The viscosity of non-Newtonian behavior is described by a power law shown in Equation (20).

$$
\mu=\left\{\begin{array}{c}
0.07, \gamma \leq 1 \\
0.07 \gamma^{n-1}, \gamma>1
\end{array}\right.
$$

Figure 16 compares the temperature and the velocity fields at $\tau=11,500$ among cases 7 to 9 which correspond to the rolling case, and Figure 17 compares those among cases 10 to 12 which correspond to the static case. It was found that with these rolling cases that the stronger the non-Newtonian behavior (lower $n$ ), the faster the temperature drop.

Obviously, as $n$ increases the average temperature becomes higher. In case 7 , the crude oil flow velocity field is slightly stronger. Comparison of average temperature drop also validates this conclusion. However, it can be found in both Figures 16 and 18 that different non-Newtonian behavior does not induce obvious differences in the thermal process for the static case.

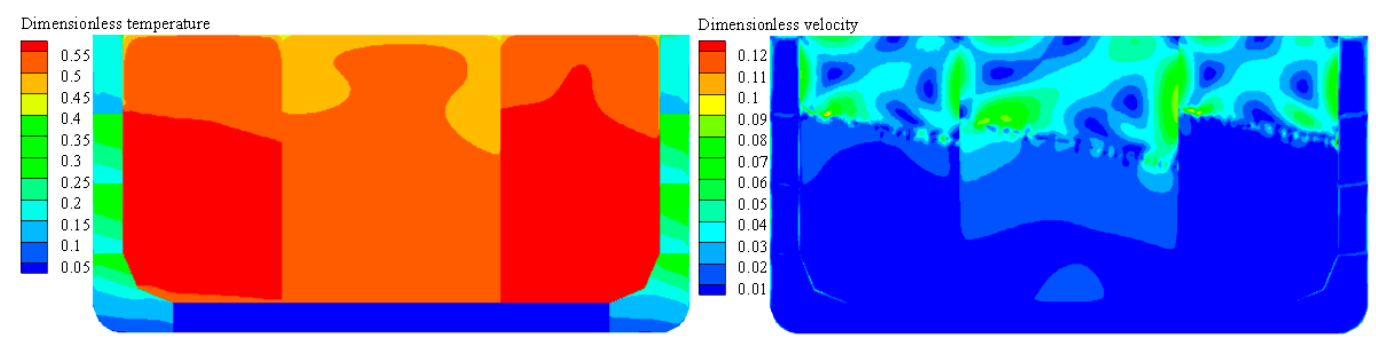

(a) Case 7

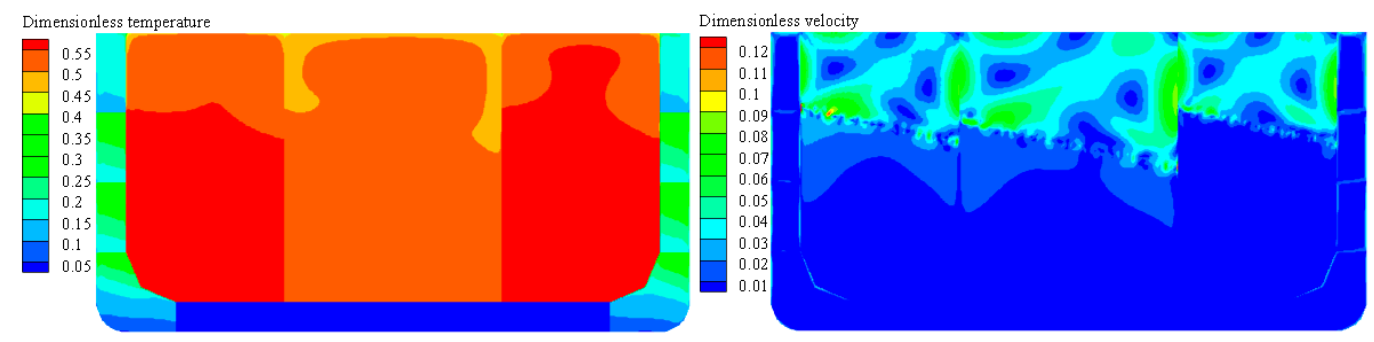

(b) Case 8

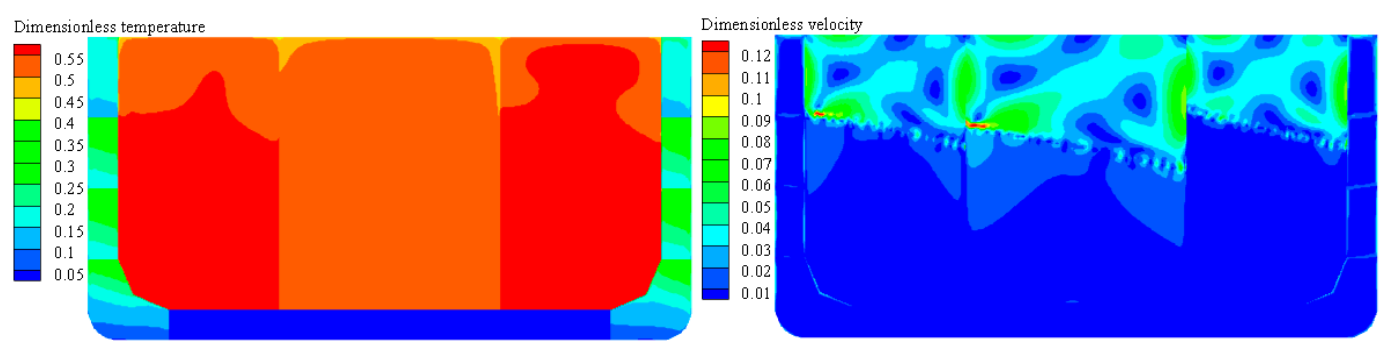

(c) Case 9

Figure 16. The temperature field and the velocity field (from top to bottom, respectively, are cases 7,8 , and 9): (a) Case 7, (b) Case 8, (c) Case 9. 

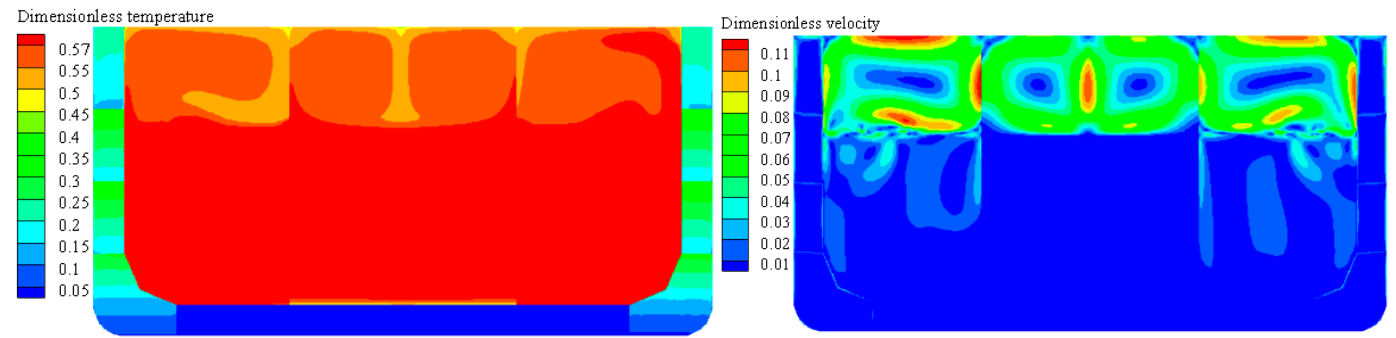

(a) Case 10

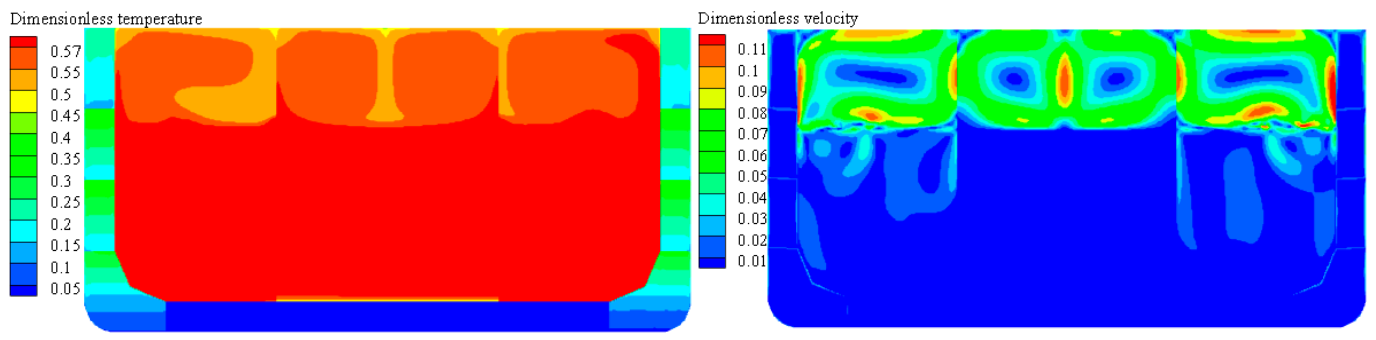

(b) Case 11

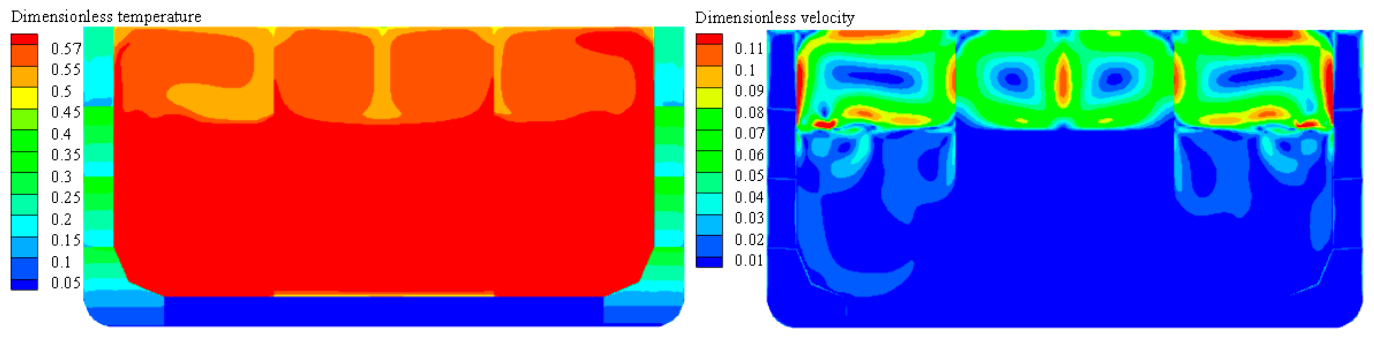

(c) Case 12

Figure 17. The temperature field and the velocity field (from top to bottom, respectively, are cases 10, 11, and 12): (a) Case 10, (b) Case 11, (c) Case 12.

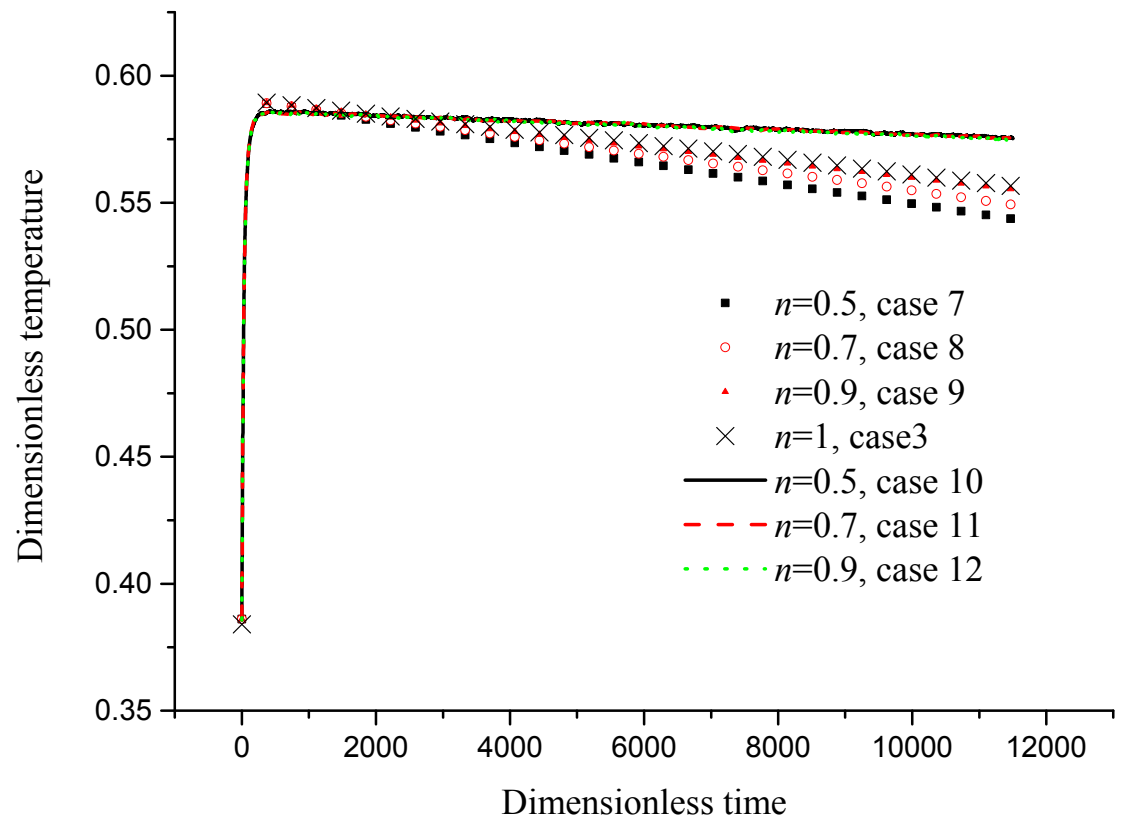

Figure 18. Comparison of temperature drop among case 2 and cases 7-12. 


\section{Conclusions}

In this paper, the effects of $R i, \omega^{*}$, and non-Newtonian behavior on the thermal-hydraulic process of crude oil subjected to a rolling motion were investigated numerically. The following conclusions were obtained with the designed cases:

(1) A rolling motion induces mixed convection, which intensifies the heat transfer depending on the Richardson number $R i$; the lower the $R i$, the faster the heat transfer.

(2) For a large-size geometry model, such as an oil tanker, the rotation-strength number $\omega^{*}$ has a negative effect on heat transfer, i.e., the larger the $\omega^{*}$, the weaker the heat transfer, since $\omega^{*}$ is dominated by geometry size.

(3) The total effect of the rolling motion on thermal-hydraulic behavior is determined by the combined effect of $R i$ and $\omega^{*}$, the effect of their combination may be different for small-size cases and large-size cases.

(4) Non-Newtonian behavior plays an important role in thermal-hydraulic process of crude oil in rolling cases, and a smaller flow-behavior index corresponds to stronger heat transfer; however its influence in the static case is weak.

Author Contributions: G.Y. directed the research and revised the paper writing. S.J. analyzed the data and wrote the original manuscript. Y.G. carried out the numerical simulation.

Funding: This research was funded by National Science Foundation of China (No. 51606117), and China Postdoctoral Science Foundation funded project (Nos. 2017M621473 and 2018T110396).

Conflicts of Interest: The authors declare no conflict of interest.

\section{References}

1. Wawrzyński, W. Area of the unstable solution of rolling equation-jumps of the oscillations amplitude. J. KONES 2018, 3, 489-496.

2. Hiroharu, K. Effects of Rolling on the Heat Transfer from Cango Oils of Tankers. J. Jpn. Soc. Naval Archit. Ocean Eng. 2009, 1969, 421-430.

3. Yu, G.J.; Yang, Q.L.; Dai, B.; Fu, Z.G.; Lin, D.L. Numerical Study on the Characteristic of Temperature Drop of Crude Oil in a Model Oil Tanker Subjected to Oscillating Motion. Energies 2018, 11, 1229. [CrossRef]

4. Doerffer, S.; Mikielewicz, J. The influence of oscillations on natural convection in ship tanks. Int. J. Heat Fluid Flow 1986, 7, 49-60. [CrossRef]

5. Akagi, S.; Kato, H. Numerical analysis of mixed convection heat transfer of a high viscosity fluid in a rectangular tank with rolling motion. Int. J. Heat Mass Transf. 1987, 30, 2423-2432. [CrossRef]

6. Tan, S.C.; Su, G.H.; Gao, P.Z. Experimental and theoretical study on single-phase natural circulation flow and heat transfer under rolling motion condition. Appl. Therm. Eng. 2009, 29, 3160-3168. [CrossRef]

7. Tan, S.C.; Su, G.H.; Gao, P.Z. Heat transfer model of single-phase natural circulation flow under a rolling motion condition. Nucl. Eng. Des. 2009, 239, 2212-2216.

8. Yu, S.; Yan, C.; Wang, J.; Cao, X.; Zhu, Z.; Wang, C. Experimental and numerical study on fluctuations and distributions of fluid temperature under rolling motion conditions. Ann. Nucl. Energy 2017, 110, 384-399. [CrossRef]

9. Liu, D.; Tian, W.; Xi, M.; Chen, R.; Qiu, S.; Su, G.H. Study on safety boundary of flow instability and CHF for parallel channels in motion. Nucl. Eng. Des. 2018, 335, 219-230. [CrossRef]

10. Wu, P.; Shan, J.; Xiang, X.; Zhang, B.; Gou, J.; Zhang, B. The development and application of a sub-channel code in ocean environment. Ann. Nucl. Energy 2016, 95, 12-22. [CrossRef]

11. Xing, D.; Yan, C.; Sun, L. Flow fluctuation behaviors of single-phase forced circulation under rolling conditions. Ocean Eng. 2014, 82, 115-122. [CrossRef]

12. Hu, Z.Q.; Wang, S.Y.; Chen, G.; Chai, S.H.; Jin, Y.T. The effects of LNG-tank sloshing on the global motions of FLNG system. Int. J. Nav. Archit. Ocean 2017, 9, 114-125. [CrossRef]

13. Grotle, E.L.; Æsøy, V. Dynamic modelling of the thermal response enhanced by sloshing in marine LNG fuel tanks. Appl. Therm. Eng. 2018, 135, 512-520. [CrossRef] 
14. Jiang, S.C.; Teng, B.; Bai, W.; Gou, Y. Numerical simulation of coupling effect between ship motion and liquid sloshing under wave action. Ocean Eng. 2015, 108, 140-154. [CrossRef]

15. Cercos-Pita, J.L.; Bulian, G.; Pérez-Rojas, L.; Francescutto, A. Coupled simulation of nonlinear ship motions and a free surface tank. Ocean Eng. 2016, 120, 281-288. [CrossRef]

16. Yang, S. Heat Transfer, 4th ed.; Higher Education Press: Beijing, China, 2006.

17. Olinde, R. Des lois géometriques qui regissent les déplacements d'un systéme solide dans l'espace, et de la variation des coordonnées provenant de ces déplacement considérées indépendant des causes qui peuvent les produire. J. Math. Pures Appl. 1840, 5, 380-440.

18. Brackbill, J.U.; Kothe, D.B.; Zemach, C. A continuum method for modeling surface tension. J. Comput. Phys. 1992, 100, 335-354. [CrossRef]

19. Ker, Y.T.; Lin, T.F. A combined numerical and experimental study of air convection in a differentially heated rotating cubic cavity. Int. J. Heat. Mass. Trans. 1996, 39, 3193-3210. [CrossRef]

20. Patankar, S.V. Numerical Heat Transfer and Fluid Flow; Hemisphere Publishing: Washington, DC, USA, 1980.

21. Tso, C.P.; Jin, L.F.; Tou, S.K.W. Numerical Segregation of the Effects of Body Forces in a Rotating, Differentially Heated Enclosure. Numer. Heat. Trans. A-Appl. 2007, 51, 85-107. [CrossRef]

22. Saleh, H.; Hashim, I. Numerical Analysis of Nanofluids in Differentially Heated Enclosure Undergoing Orthogonal Rotation. Adv. Math. Phys. 2014, 2014,1-11. [CrossRef]

(C) 2019 by the authors. Licensee MDPI, Basel, Switzerland. This article is an open access article distributed under the terms and conditions of the Creative Commons Attribution (CC BY) license (http://creativecommons.org/licenses/by/4.0/). 